\title{
USP9X can be used to predict pancreatic cancer outcomes
}

The authors of a recent Nature paper suggest that the $\mathrm{X}$-linked deubiquitinase USP9X is an important tumour suppressor gene in pancreatic ductal adenocarcinoma that could be used to determine prognosis and to improve therapies in patients with this cancer.

As previous models of pancreatic ductal preneoplasia progressed to invasive cancer slowly, the researchers decided to search for genes that promoted pancreatic cancer. Several different methods were explored before the researchers decided on a technique that involved transposon mutagenesis to create a mouse model of pancreatic ductal adenocarcinoma.

"Transposons are mobile genetic elements that can be liberated specifically in somatic cells, enabling the whole genome to become mutagenized," explains corresponding author David Tuveson (Cambridge Research Institute, Cancer Research UK). "Cells that carry new mutations and have a selective growth or survival advantage will accumulate and form an invasive pancreatic cancer. The DNA from the resulting tumour is simply analyzed for the insertion sites of the transposons, and genes that are disrupted are identified following statistical analysis."
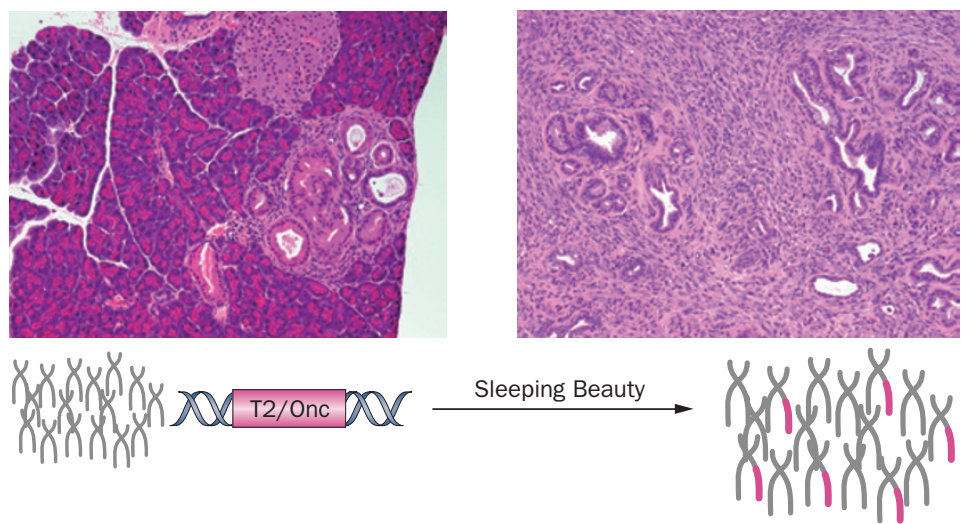

Sleeping Beauty transposon-mediated insertional mutagenesis was used to identify candidate genes that could be involved in the progression from pancreatic ductal preneoplasia (left) to pancreatic ductal adenocarcinoma (right). Image produced in consultation with P. A. Pérez-Mancera.

\begin{abstract}
Usp $9 x$ was the most commonly mutated gene in this mouse model of pancreatic ductal adenocarcinoma; the researchers found that Usp9x was deactivated in 50\% of the tumours.

These findings were confirmed in three cohorts of patients with pancreatic ductal adenocarcinoma. Low expression of USP9X was correlated with poor survival in 100 Australian patients undergoing surgery. In 42 American patients, expression of the USP9X protein was inversely correlated with widespread metastasis. Analysis of tumour specimens from 404 German patients revealed that USP9X expression was lower in tumour tissue than in normal pancreatic tissue, and confirmed that low expression levels correlated with poor outcomes.

"Currently, we are continuing to characterize the USP9X pathway in detail," says Tuveson. "Additionally, our work suggests that patients with loss of USP9X might benefit from drugs that can reactivate epigenetically silenced genes."
\end{abstract}

\section{Claire Greenhill}

Original article Pérez-Mancera, P. A. et al. The deubiquitinase USP9X suppresses pancreatic ductal adenocarcinoma. Nature doi:10.1038/nature11114

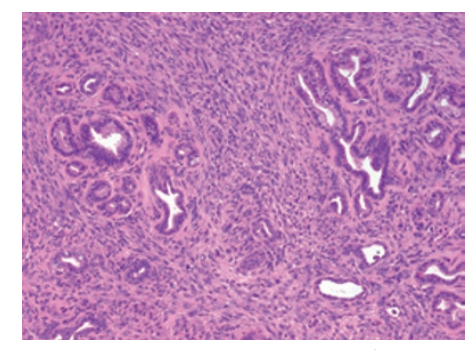

